\title{
Effects of seabed reflection on the accuracy of acoustic holography test analysis
}

\author{
Dengyuan Mo 1,a, Qidou Zhou ${ }^{2}$, Shaochun Ding ${ }^{3}$, Xiaojun Lve ${ }^{2}$ \\ ${ }^{1}$ Naval University of Engineering, College of Power Engineering, 430033, Wuhan, Province Hubei, China \\ ${ }^{2}$ Naval University of Engineering, Faculty of Naval Vessel Engineering, 430033, Wuhan, Province Hubei, China \\ ${ }^{3}$ Naval University of Engineering, Department of Science R\&D, 430033, Wuhan, Province Hubei, China
}

Keywords: Acoustic test; Half-space; Seabed reflection; Error analysis

\begin{abstract}
In this paper, the relevant research work is carried out about seabed sound reflection. It has influences on the test results of a real-boat test based on the acoustic holographic technology. The effects of seabed reflection on underwater acoustic test is analyzed, through simulating sound field of free field and semi-free field are seabed with different absorption capacity. By comparing the amplitude and phase relative errors under the free field and halffree field with different seabed, the seabed selection criteria for the acoustic test is determined, and the upper frequency limit is discussed. The basis for sitting sea trials used to do real-boat test based on the acoustic holographic technology and optimizing test program are proposed.
\end{abstract}

\section{Introduction}

NAH (NAH: near acoustic holography) technologic was firstly proposed and applied to the submarine radiated noise tests by Williams [1-2]. Due to the relatively low cost and a high precision, Australian Defence Science and Technology Organisation and the Naval Undersea Technology Center jointly launched submarine test based on the NAH in mooring state[3]. The test results only had the measurement error $2 \mathrm{~dB}$ comparing with the test which costs about 250 million.

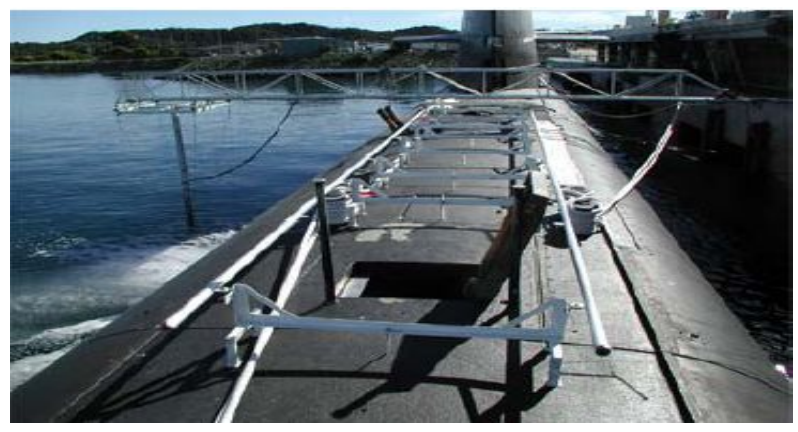

Figure 1. The physical map of NAH test system of the USA and Australia

Fig. 1 is a kind of real-boat test system about NAH in USA and Australia. 
As is known, submarine radiated noise testing has extremely high requirements for marine environment, so sitting is very important. The literatures [3-5] summed that the shelter water area with a better and stable meteorological conditions must be considered in the first, and the water area is wider and the depth meets the testing requirements. US has three major testing ground for the sound of water: Atlantic Underwater Test and Evaluation Center, Southeast Alaska Acoustic Proving Ground Water and The unmanned submarine Daldry Lake Proving Ground. These testing grounds with good water environment and less outside interference are very suitable for submarine radiated noise testing. However our natural marine environment and the condition of wharf or port is not good, the seabed sound reflection must have a influence on the test accuracy. In addition, the experience of underwater acoustic holography testing in the marine environment is a few and few studies can be referred. So, In order to promote the application of acoustic holography technique in submarine radiated noise test, it is necessary to study the effect of seabed sound reflection on the accuracy of acoustic holography test. And it is also good for improving the accuracy of the submarine radiated noise test.

In this paper, based on the image method and theory of acoustic holography in the half-field[6-8], the simulations of the effects of seabed reflection on underwater acoustic test are analysed. Both the shallow seawater and the sound source which is close to the seabed are considered under the simulation condition. The study has an important project implication for the real boat test based on the nah to sit sea trials and optimize test program.

\section{The theory of the method of half-space acoustic holography}

The planer near field acoustic holography is usually used to the free space sound field. However, when seawater is shallow and the sound source is close to the seabed, sound wave will have a reflection at the seabed boundary interface, in other words, sound waves spread in a half-field not free-filed. And sound source signal that is received by the system is made up of direct sound and reflection sound. In such a measurement environment, the theory of nah can't be simply applied to analyse sound field.

\subsection{Calculate distribution of sound field in the half-space by the method of mirror image}

In the free space sound field, acoustic potential function should satisfy the wave equation (1):

$$
\nabla^{2} \psi+k^{2} \psi=0
$$

here, $k=\frac{\omega}{c}$ 。

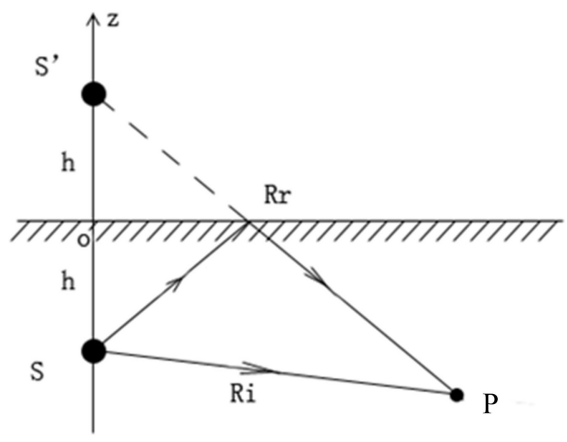

Figure 2. The principle of the method of mirror imaging 
The medium is a half-free sound field if it has an interface. When the reflection is total reflection on the interface, the acoustic potential function $\psi$ should satisfy the following condition:

$$
\frac{\partial \psi}{\partial z}=0
$$

The method of mirror imaging is commonly used to solve the half-space. The principle of it is shown in fig2.

$\mathrm{S}$ is the real sound source under the medium interface. Assuming there is a virtual sound source $\mathrm{S}$ ' which is symmetrical with the real sound source about the medium interface. the sound strength of measuring point $\mathrm{P}$ is equal to the sum sound strength of $\mathrm{S}$ and $\mathrm{S}$ '. Assume that the synthetic sound field is made up of the sound field of the real sound source and a virtual sound source which is got by making a specular reflection of the real sound source on the medium interface, so the equation of synthetic field is as follow:

$$
\psi=\frac{e^{j k R_{i}}}{R_{i}}+\frac{e^{j k R_{r}}}{R_{r}}
$$

$R_{i}$ is the distance between the real sound source $\mathrm{S}$ and the measuring point $\mathrm{P} . R_{r}$ is the distance between the virtual sound source $S$ ' and the measuring point $P$.

\subsection{Solve seabed reflection by the method of half-space NAH}

Because the measurement signal in the half-space is the synthesis of the direct and specular reflection sound. And sound press is a scalar. So it is difficult to directly separate the direct sound waves from measurement-sound-press on holographic plane. It is difficult to use the conventional technology of PNAH to directly reconstruct sound filed of sound source plane. And it is supposed to be considered the characteristics of the half-space sound field for the holography analysis.

Because the interface features can divide into an absolutely soft interface, an absolutely hard interface or impedance interfaces. According to the principle of the mirror, the measured data on the measured surface can be extended to the other side to get the holography surface. Though the above step, the extend holography surface which is symmetry with the original holography surface about the interface. And then it is need to do a symmetry-integral sound filed of the holography surface to get the reconstruction surface. At last, it is necessary to remove the virtual sound source of the reconstruction surface to get the final reconstruction results. The transformation progress of half- space NAH is shown in Fig.3.

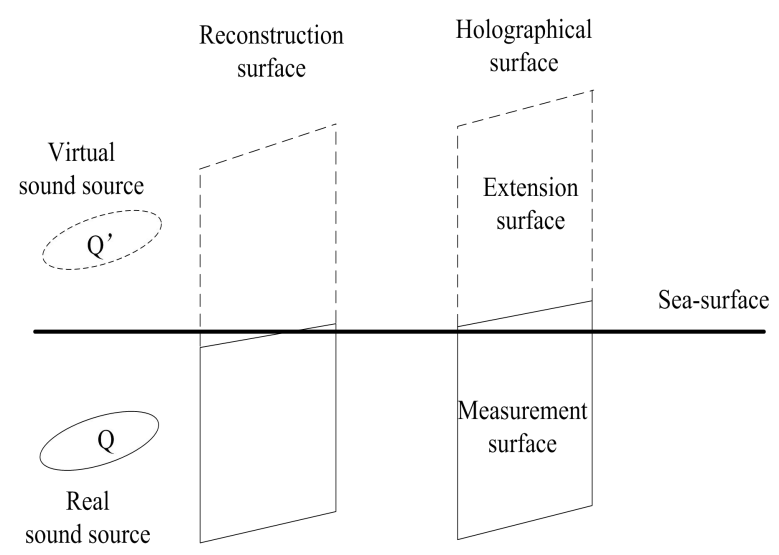

Figure 3. The transformation diagram of half-field NAH 


\section{Simulation}

In this paper, the corresponding simulation work is carried out for a specific test sea conditions, in which the water depth is $10 \mathrm{~m}$. The maximum value of sound source position of the measured structure is about $8 \mathrm{~m}$ under water. And minimum source-level is about $120 \mathrm{~dB}$.

The sound absorption of seawater is not considered in the simulation. But the role of seabed sound absorption is considered. The seabed is approximately flat, and sound field space is approximately half-free field. Three pulse source balls are used which are $0.1 \mathrm{~m}$ in radius and the same height $1 \mathrm{~m}$ from the seabed is used. The sound source spacing is $1 \mathrm{~m}$, and the sound stream level is $120 \mathrm{~dB}$. Seawater density is $1000 \mathrm{~g} / \mathrm{cm}^{3}$, water velocity of sound is $1500 \mathrm{~m} / \mathrm{s}$. And the distance is $\lambda / 3$ between the sound source and the holographic surface. The distance is $\lambda / 20$ between the sound source and the reconstruction surface The size of holographic surface is $6 \mathrm{~m} \times 6 \mathrm{~m}$, and the grid size is $\lambda / 10$. The frequency of simulation include $20 \mathrm{~Hz}, 40 \mathrm{~Hz}, 60 \mathrm{~Hz}, 100 \mathrm{~Hz}, 150 \mathrm{~Hz}, 200 \mathrm{~Hz}, 250 \mathrm{~Hz}, 300 \mathrm{~Hz}, 400 \mathrm{~Hz}$, $500 \mathrm{~Hz}, 700 \mathrm{~Hz}, 1000 \mathrm{~Hz}$.

Amplitude and phase errors of nah test are shown in Fig. 4 and Fig.5 under different conditions of the seabed reflection. In which, B represents the seabed absorption capacity. It could be seen that B is larger, the errors of amplitude and phase are smaller. Because the absorption quantity of the seabed is about $8 \mathrm{~dB}$, it is necessary to choose a larger absorption quantity seabed for the Nah tests in the same sea conditions. In addition, the frequency is higher, the errors of amplitude and phase are larger. So, a reasonable upper limit of the frequency analysis based on test conditions should be determined in the actual analysis.

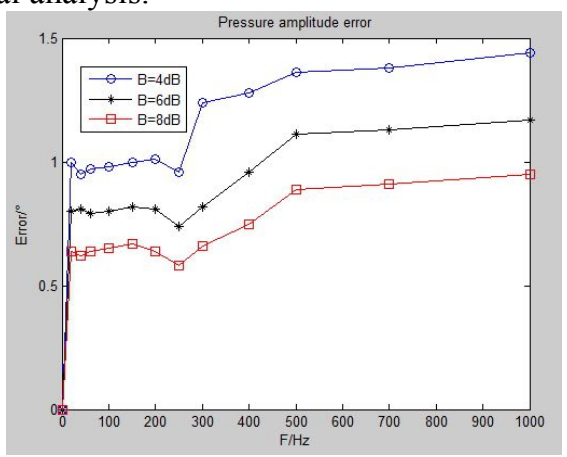

Figure 4. The seabed reflection on reconstruction sound amplitude

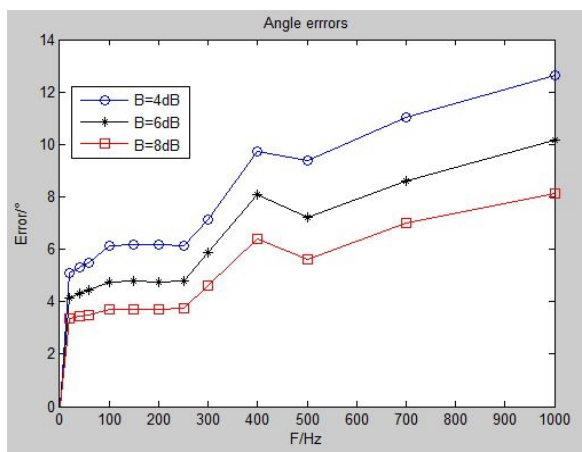

Figure 5. The seabed reflection on reconstruction sound phase

The reconstruction results in the free-field and the half-field with the reflection loss of $8 \mathrm{~dB}$ are shown in the Fig. 6 and Fig.7. It is obvious that when reflection loss is $8 \mathrm{~dB}$ the reconstruction phase distribution is almost same. And the reconstruction amplitude distribution is slightly different. Both the reconstruction phase and amplitude results can better reproduce the sound source information, which basically meet the requirements of engineering applications.
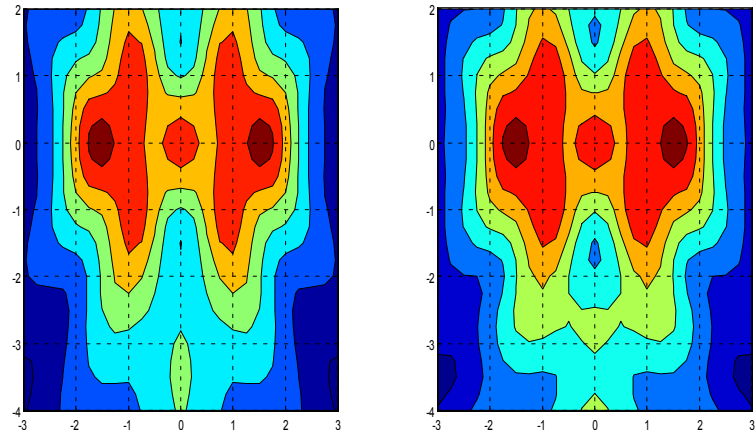

Figure 6. Under semi-free field with seabed reflection loss of $8 \mathrm{~dB}$ and free field at $\mathrm{f}=1000 \mathrm{Hzsound}$ amplitudes comparison chart 

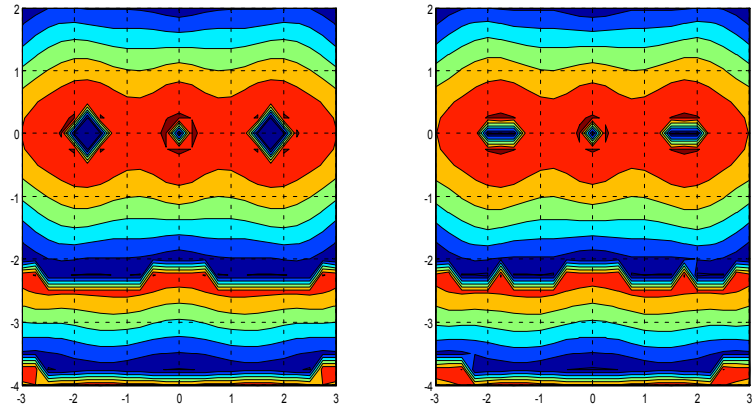

Figure 7. Under semi-free field with seabed reflection loss of $8 \mathrm{~dB}$ and free field at $\mathrm{f}=1000 \mathrm{hz}$,sound phase comparison chart

In summary, when the analysis frequency is not lower than $1000 \mathrm{~Hz}$, it is a better choice to select a sediment location to do an acoustic holography test.

\section{Conclusions}

In this paper, the relevant research is carried out about the seabed sound reflection that has influences for the test results of the real-boat test. Based on the image method and theory of acoustic holography in the half-field, the simulations of the effects of seabed reflection on underwater acoustic test are analysed. The researches show that the absorption capacity is larger, the test accuracy is higher. And the frequency of the sound source is higher, the test error is larger. The conclusion is important for the real-boat test based on the NVH to sit sea trials and optimize test program.

\section{References}

1. Williams E G,Houston B H, Bucaro J A. Broadband Near-field Acoustical Holography for Vibrating Cylinders [J]. J.Acoust.Soc.Am., Vol.85 No.2: 674-679. (1989)

2. Williams E G, Houston B H, Peter C Herdic. Interior Near-field Acoustical Holography in Flight [J]. J.Acoust.Soc.Am, ,Vol.108 No.4 :1451-1463. (2000)

3. Liu Xing-zhang,etc.Development prospect and revelation of submarine underwater noise test ground of US.Navy.[J].Ship Science and Technology,Vol.33 No.2: 140-143. (2011)

4. Wang Da-hai,Liu Xing-zhang,etc..Analysis of facilities in the Atlantic undersea test and evaluation center[J].Ship Science and Technology, Vol.33 No.10:140-143. (2011)

5. QIU Wei-hai, LIU Wen-shuai, WANG Xiu-bo, etc.. Technology for foreign ship radiated noise measurement, Vol.33 No.4 : 147-150. (2011)

6. ZHANG Ruo-yu,Cylinder Near-field Acoustical Holography in Half-space and Its Visualization [D](Master's degree), Harbin Engineering University : 13-55. (2009)

7. LIU Li-hua,Investigation on Technique of Underwater Noise Source Identification by NAH in Semi-Space [D](Master's degree), Harbin Engineering University:10-21. (2008)

8. CHEN Xin-zhao, BI Chuan-xing,LI Wei-bing, YU Fei, etc.. Novel near-field acoustic holography based on the distributed source boundary point method[J]. Hefei University of Technology (Natural Science), Vol.28 No.9: 961-966. (2005) 